\title{
Analysis and Evaluation of a Quasi-Passive Lower Limb Exoskeleton for Gait Rehabilitation
}

\author{
Niaam Kh. Al-Hayali* \\ Jumaa S. Chiad*** \\ Somer M. Nacy** \\ O. Hussein**** \\ ***Department of Biomedical Engineering/Alkhwarizmi College of Engineering/ \\ University of Baghdad/Baghdad - Iraq \\ *** Department of Mechanical Engineering/College of Engineering/Al-Nahrain University/ Baghdad - Iraq \\ **** Department of Automated Manufacturing Engineering/Alkhwarizmi College of Engineering/ \\ University of Baghdad/ Baghdad - Iraq \\ *Email: neaam@kecbu.uobaghdad.edu.iq \\ **Email: somernacy@googlemail.com \\ ***Email: jumaachiad@gmail.com \\ ****Email: omar.hussein@kecbu.uobaghdad.edu.iq
}

(Received 4 August 2021; Accepted 20 December 2021)

https://doi.org/10.22153/kej.2021.12.007

\begin{abstract}
Lower extremity exoskeletons can assist with performing particular functions such as gait assistance, and physical therapy support for subjects who have lost the ability to walk. This paper presents the analysis and evaluation of lightweight and adjustable two degrees of freedom, quasi-passive lower limb device to improve gait rehabilitation. The exoskeleton consists of a high torque DC motor mounted on a metal plate above the hip joint, and a link that transmits assistance torque from the motor to the thigh. The knee joint is passively actuated by spring installed parallel with the joint. The action of the passive component (spring) is combined with mechanical output of the motor to provide a good control on the designed exoskeleton while walking. The results show that muscles' efforts on both the front and the back sides of the user's leg were decreased when walking using the exoskeleton with the motor and spring.
\end{abstract}

Keywords: Exoskeleton, lower limb, gait cycle, quasi-passive, rehabilitation.

\section{Introduction}

Assistive exoskeletons for healthcare like rehabilitation and gait assistance has been widely developed [1][2][3]. Lower limb exoskeletons are defined as wearable devices that are worn by a human and work in concert with the wearer's movements [4][5]. Lower limb exoskeletons can be used to aid the rehabilitation training by providing functional motion assistance for people with neurological and age related disorders [3][6][7][8]. During manual rehabilitation training, the training time is limited to the therapist. With this limitation of time, the gait style of the patient is not reproducible and thus the whole therapy is not optimal [9][10]. Rehabilitation exoskeletons allow normal and symmetrical walking style with raising the period and intensity of the practice sessions [11][12]. A number of lower limb exoskeletons and their features for rehabilitation have been presented by researchers. In 2003, Hiroaki Kawamoto et al developed HAL which is integrated with the human and assists proper power for lower extremity of patients with a gait disorder. The exoskeleton is actuated by electrical motor and 
controlled based on EMG signals [13][14]. In 2004, an over ground lower limb exoskeleton BLEEX from University of California-Berkeley was designed for people's rehabilitation and assistance. The exoskeleton is actuated by three hydraulic cylinders for each leg, and its control based on feet pressure signals [5][15][16]. In 2006, EXPOS was developed by Sogang University for patients and elderly people. EXPOS integrated an automatic platform as caster walker to retain the balance during walking. The device is pneumatically actuated, and its control by the neural network theory [2][9]. The REX exoskeleton by REX Bionics was developed in 2007 for subjects with spinal cord injury (SCI) [17] which is able to self- balance during motion. REX is actuated by an electrical motor, and it is the only completely self-supporting (i.e. handfree), independently controlled exoskeleton available among both the commercial and research prototypes [18][19]. In 2007, ALEX developed by Sai K. Banala et al, which is a powered orthosis device used for gait training of subjects with walking disorders. The exoskeleton has linear actuators at hip and knee joints [20][21], it is also instrumented with force-field controller [22]. In 2013, Ekso Bionics [23] developed an exoskeleton designed for gait rehabilitation and assistance purposes, named "Ekso GT Exoskeleton". This design has been developed as a powered over ground exoskeleton containing a hydraulic actuator. The assistance level presented by Ekso device can be adjusted according to user's need [8]. In 2020, Y. M. Pirjade et al. designed and fabricated eight degrees of freedom lower extremity exoskeleton for gait training. The lower extremity hybrid exoskeleton works on a pre-defined walking gait cycle and is driven by electric actuators and mechanical springs. The exoskeleton has electric actuators at the hip and knee joints which provide assistive torque at the joints. The ankle joint of the device is passively actuated by a spring [24]. This paper aims to analyze and develop a new lower limb exoskeleton for gait rehabilitation to assist patients in their walking. Since the above mentioned exoskeletons are active (powered) devices and need more sophisticated instruments to be controlled and according to the limitations of the power-to-weight ratio that appears in such exoskeletons, the presented exoskeleton is quasipassive which is lightweight and require very low power to work.

\section{Materials and Methods}

The assistive exoskeleton presented in this work is proposed to serve both able-bodied and subjects with lower limb weakness by reducing their lower limb muscles effort during walking.

\subsection{Clinical Gait Cycle Analysis}

Clinical gait analysis is important, not only to build a mathematical model, but also to improve the design of exoskeletons. The AMIT biomechanics force plate model OR6-7 was used for the measurement of the ground reaction forces (GRFs) of the right lower limb of the healthy participant. AMIT's BioAnalysis Software is a completely- featured biomechanics analysis to analyze the biomechanics of human gait cycle. The AMTI's NetForce data gathering software can be interfaced directly with the AMIT's BioAnalysis program for analyzing and processing the data. The vertical and horizontal GRFs, which are acted on the undersurface of a subject's right foot throughout the walking cycle were calculated. Simultaneously, a camera was used to measure the motion tracking in order to be analyzed and evaluated by Kinovea to calculate the angles of the hip and knee joints throughout a complete gait cycle.

\subsection{Mathematical Model of Human Lower Limb}

In order to choose the appropriate motor to be used in the presented design, a mathematical model for the lower extremity of the human body throughout a full and normal gait cycle was built to calculate the hip joint torque. The following mathematical analysis for human lower limb is based on measuring the kinematics of the human body parts complemented with the GRFs measurement. The inertia forces were neglected in this analysis, thus the presented analysis was considered as a quasi-static analysis.. Consider the right lower limb, the hip joint torque can be calculated throughout different phases of the gait cycle as illustrated below. The angle between the foot and the leg is not considered in this research, the foot is considered as a rigid body in the human model.

Where, $\mathrm{m}_{1}$ : is the thigh mass, $\mathrm{m}_{2}$ : is the shank mass, and $m_{3}$ : is the foot mass. The locations of these masses on the leg are approximate and not accurate. $1_{1}$ : is the thigh length, and $l_{2}$ : is the shank 
length. $F_{x}$ : is the horizontal GRF, $F_{y}$ : is the vertical GRF. and $T_{1}$ : is the hip joint torque.

1- Stance Phase - $1^{\text {st }}$ double support: Throughout the $1^{\text {st }}$ double support phase, the hip and knee joints are both in the condition of flexion as shown in figure 1. The hip joint so as the knee joint are both positive and mainly the hip joint angle is less than the knee joint.

Taking moment at hip joint $\left(\mathrm{T}_{1}\right)$ :

$\mathrm{T}_{1}+\mathrm{m}_{1^{*}} \mathrm{~g}\left[\left(\mathrm{I}_{1} / 2\right) \sin \theta_{1}\right]+\mathrm{m}_{2^{*}} \mathrm{~g}\left[\left(\mathrm{I}_{2} / 2\right) \sin \left(\theta_{1^{-}}\right.\right.$

$\left.\left.\theta_{2}\right)+l_{1} \sin \theta_{1}\right]+m_{3^{*}} g\left[l_{2} \sin \left(\theta_{1}-\theta_{2}\right)+l_{1} \sin \theta_{1}\right]=$

$F_{x}\left[l_{2} \cos \left(\theta_{1}-\theta_{2}\right)+I_{1} \cos \theta_{1}\right]+F_{y}\left[l_{2} \sin \left(\theta_{1}-\theta_{2}\right)+I_{1} \sin \theta_{1}\right]$

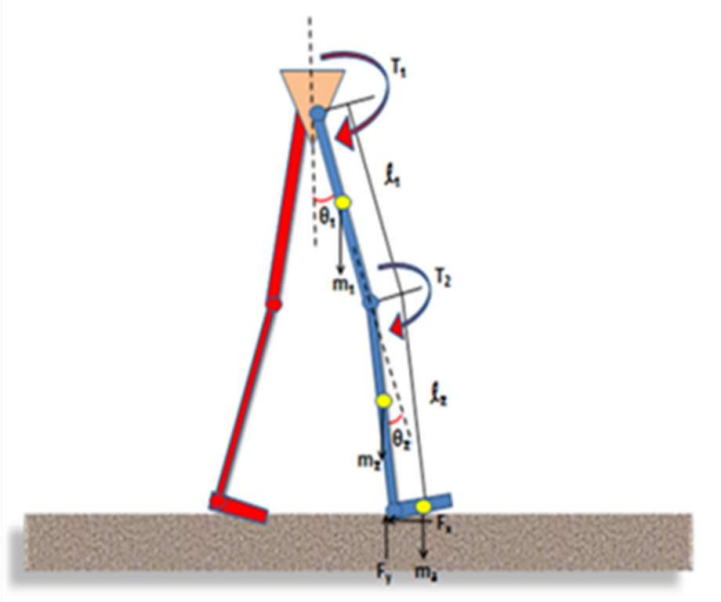

Fig. 1. $1^{\text {st }}$ double support phase.

2- Stance Phase - Single limb stance: In the case of when the hip joint is in the condition of flexion and the knee joint angle is greater than the hip joint as shown in Figure 2.

Taking moment about hip joint (T1):

$\mathrm{T} 1+\mathrm{m} 1 * \mathrm{~g}[(11 / 2) \sin \theta 1]+\mathrm{m} 2 * \mathrm{~g}[(12 / 2) \sin (\theta 1-$

$\theta 2)+11 \sin \theta 1]+\mathrm{m} 3 * \mathrm{~g}[12 \sin (\theta 1-\theta 2)+11 \sin \theta 1]=\mathrm{Fx}$

$[12 \cos (\theta 1-\theta 2)+11 \cos \theta 1] \quad+\mathrm{Fy}[12 \sin (\theta 1-$

$\theta 2)+11 \sin \theta 1]$

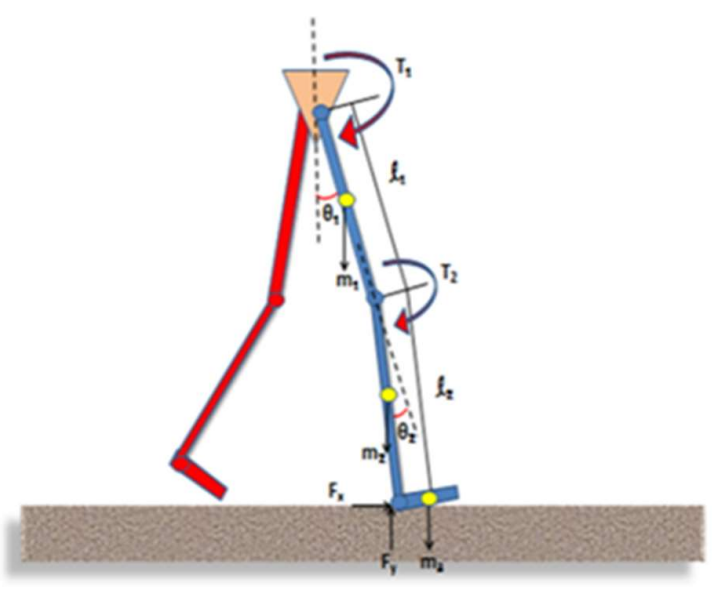

Fig. 2. Single limb stance with hip joint flexion.

3- Stance Phase - Single limb stance: In the case of when the hip joint is in the condition of extension and the knee joint angle is greater than the hip joint as shown in Figure 3.

Taking moment about hip joint (T1):

$\mathrm{T} 1=\mathrm{m} 1 * \mathrm{~g}[(11 / 2) \sin \theta 1]+\mathrm{m} 2 * \mathrm{~g}[(12 / 2) \sin (\theta 1+\theta 2)+11 \mathrm{~s}$ in $\theta 1]$

$+\mathrm{m}^{*} \mathrm{~g}[\mathrm{I} \sin (\theta 1+\theta 2)+\mid 1 \sin \theta 1]+\mathrm{Fx}[12 \cos (\theta 1+\theta 2)+11$

$\cos \theta 1]+F y[12 \sin (\theta 1+\theta 2)+\mid 1 \sin \theta 1]$

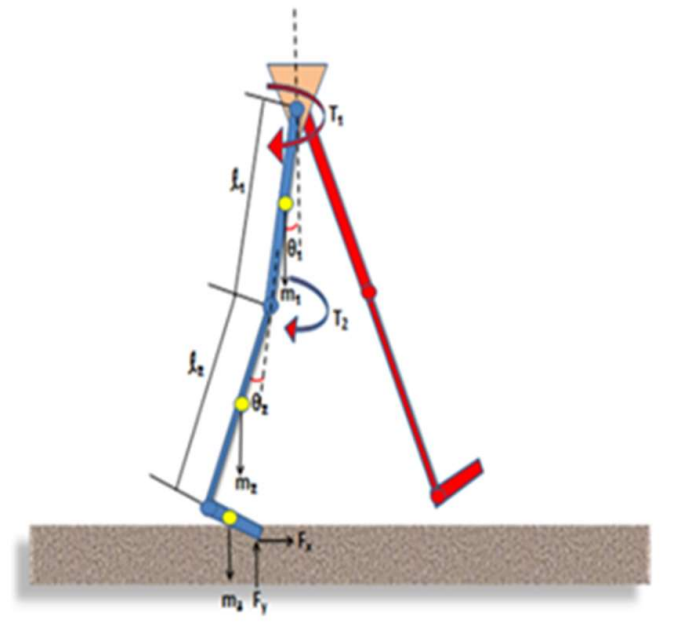

Fig. 3. Single limb stance with hip joint extension.

4- Stance Phase - $2^{\text {nd }}$ double support: Throughout the $2^{\text {nd }}$ double support phase, the hip joint is in the condition of extension with a negative joint angle, and the knee joint is in the condition of flexion with a positive joint angle as shown in Figure 4. 
Taking moment about hip joint $\left(\mathrm{T}_{1}\right)$ :

$\mathrm{m}_{1^{*}} \mathrm{~g}\left[\left(\mathrm{l}_{1} / 2\right) \sin \theta_{1}\right]+\mathrm{m}_{2^{*}} \mathrm{~g}\left[\left(\mathrm{l}_{2} / 2\right) \sin \left(\theta_{1}+\theta_{2}\right)+\mathrm{l}_{1} \sin \theta_{1}\right.$

]$+m_{3^{*}} \mathrm{~g}\left[\mathrm{l}_{2} \sin \left(\theta_{1}+\theta_{2}\right)+\mathrm{l}_{1} \sin \theta_{1}\right]+\mathrm{F}_{\mathrm{x}}$

$\left[l_{2} \cos \left(\theta_{1}+\theta_{2}\right)+l_{1} \cos \theta_{1}\right]=$

$\mathrm{F}_{\mathrm{y}}\left[\mathrm{l}_{2} \sin \left(\theta_{1}+\theta_{2}\right)+\mathrm{l}_{1} \sin \theta_{1}\right]+\mathrm{T}_{1}$

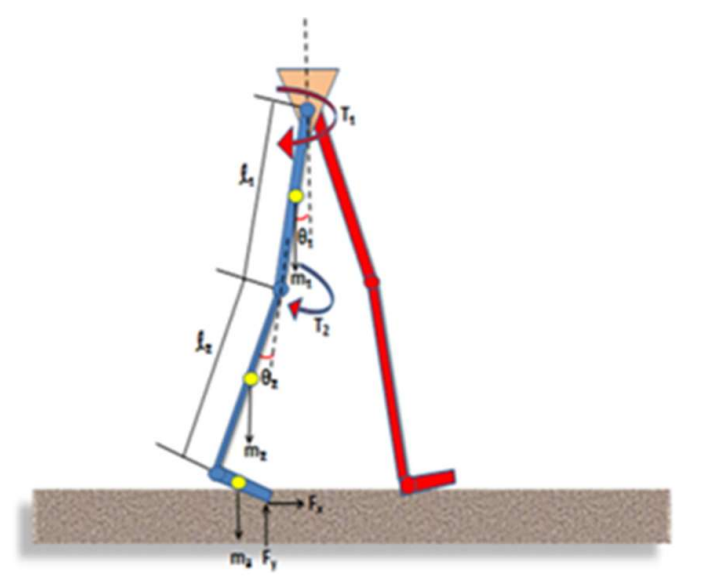

Fig. 4: $2^{\text {nd }}$ double support phase.

5- Swing phase: In the case of when the hip joint is in the condition of extension and the knee joint is in the condition of flexion as shown in Figure 5:

Taking moment about hip joint $\left(\mathrm{T}_{1}\right)$ : $\mathrm{m}_{1^{*}} \mathrm{~g}\left[\left(\mathrm{l}_{1} / 2\right) \sin \theta_{1}\right]+\mathrm{m}_{2^{*}} \mathrm{~g}\left[\left(\mathrm{l}_{2} / 2\right) \sin \left(\theta_{1}+\theta_{2}\right)+\mathrm{l}_{1} \sin \theta_{1}\right.$ ]$+m_{3^{*}} g\left[l_{2} \sin \left(\theta_{1}+\theta_{2}\right)+l_{1} \sin \theta_{1}\right]=\mathrm{T}_{1}$

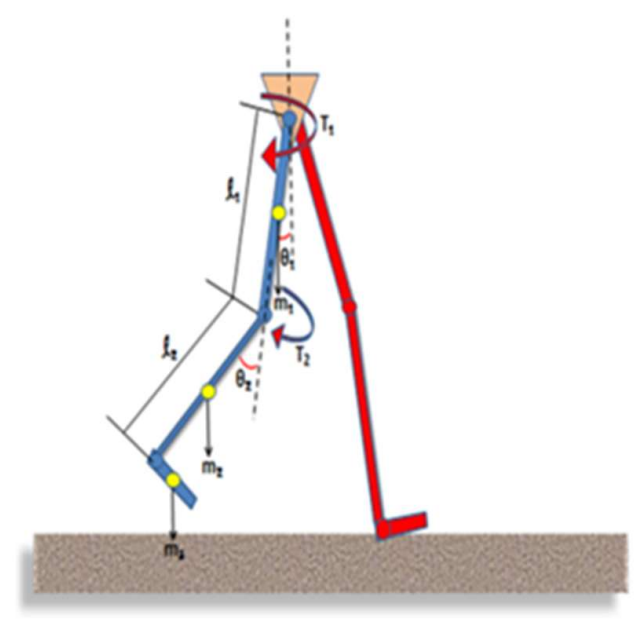

Fig. 5. Swing phase with hip joint extension and knee joint flexion.

6- Swing phase: In the case of when both joints are in the condition of flexion as shown in Figure 6.

Taking moment about hip joint $\left(\mathrm{T}_{1}\right)$ :

$$
\begin{aligned}
& \mathrm{T}_{1}= \\
& \mathrm{m}_{2^{*}} \mathrm{~g}\left[\mathrm{l}_{1} \sin \theta_{1}+\left(\mathrm{l}_{2} / 2\right) \sin \left(\theta_{1}-\theta_{2}\right)\right] \\
& \mathrm{m}_{3^{*}} \mathrm{~g}\left[\mathrm{l}_{1} \sin \theta_{1}+\mathrm{l}_{2} \sin \left(\theta_{1}-\theta_{2}\right)\right]\left[\left(\mathrm{l}_{1} / 2\right) \sin \theta_{1}\right]-
\end{aligned}
$$

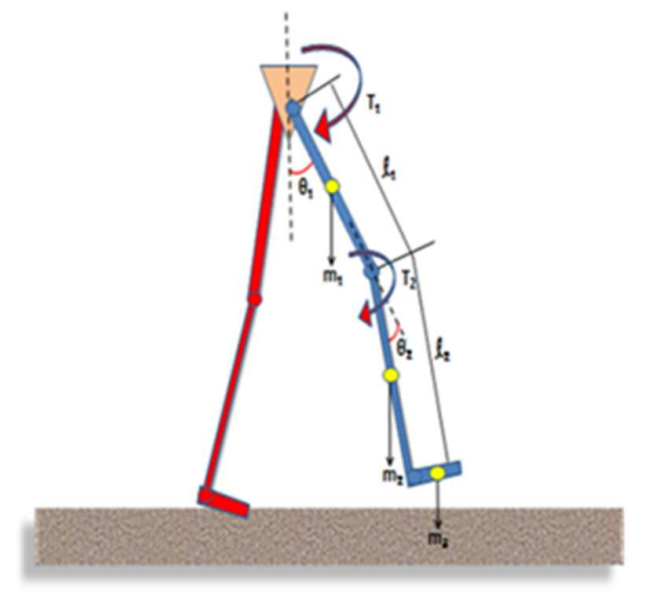

Fig. 6. Swing phase with hip and knee joints flexion.

In order to calculate the torques at lower limb hip joint, the variation of the hip and knee joint's angles, and the vertical and horizontal GRFs throughout the gait cycle and its various phases were measured to be used as an input data for Matlab program. Results reveal that the maximum calculated value of torque for the hip joint throughout the complete gait cycle is $64.702 \mathrm{~N} . \mathrm{m}$ during the stance phase.

\subsection{The Proposed Exoskeleton}

A two degree of freedom (DOF) quasi-passive lower limb exoskeleton is designed to improve gait rehabilitation. The material design and selection tried not to increase the weight of the proposed exoskeleton and its components. In addition to its light weight, the exoskeleton is developed to be adjustable to be used by different users. All the components of the exoskeleton frame were made from strong lightweight aluminum alloy with foam padding to provide good ventilation, firm support, and keep maximum comfort while use. The elements in the exoskeleton were chosen based on an analysis of the kinetics and kinematics of the human walking. The schematic and overall mechanical structure of the proposed exoskeleton is shown in figure 7. This exoskeleton consists of a high torque DC servo motor, springs, links, belts, and cuffs. The electrical circuits of the proposed exoskeleton include the motor drive and the microcontroller. The final device weights about $2.7 \mathrm{~kg}$ without the power supply. 


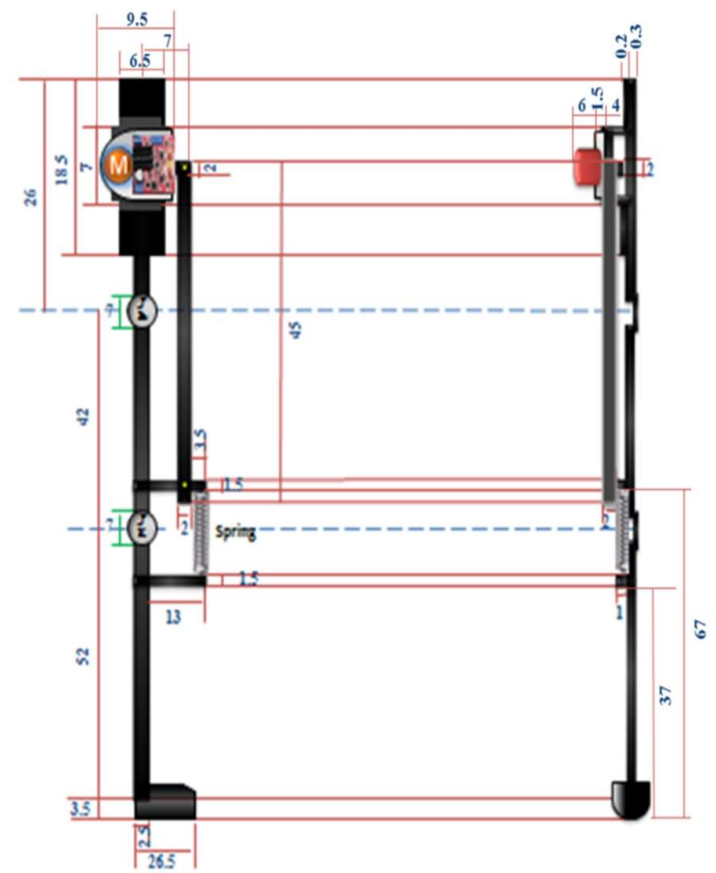

(a)

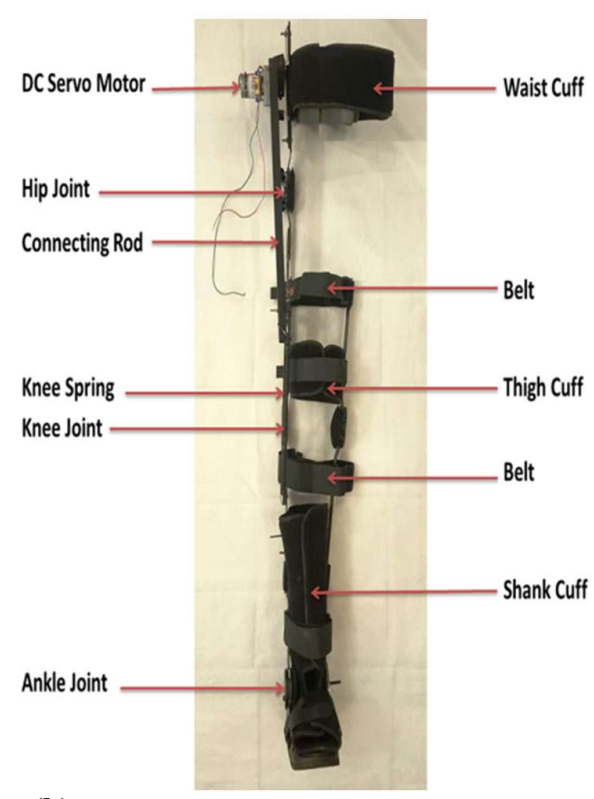

(b)

Fig.7. The proposed lower limb exoskeleton components (a) the schematic diagram of proposed Exoskeleton side and front view (all dimensions in $\mathrm{cm}$ ), (b) the overall device.

During the design of exoskeleton, an optimal set of DOFs was chosen in order to allow for a user to walk normally and safely by the exoskeleton. The hip joint has three main axes which allow movement in three degree of freedom. All axes go through the rotational center of the hip joint. The flexion/extension of the hip means moving the thigh forward/backward, adduction/abduction of the hip means sideways movement of the leg away from the body/inward movement of the leg toward the midline of the body, and lateral/medial rotation means rotating movement towards the midline/rotating movement away from the midline [25]. For the hip joint, flexion/extension was included in the exoskeleton design while the abduction/adduction and lateral/medial rotations were blocked as shown in table 1. Similarly, knee flexion/extension was included in the exoskeleton design. For the ankle joint, the joint was locked at $0^{\circ}$ angle and motions in all planes were blocked.

Table 1,

Allowed and blocked DOF of the proposed lower limb exoskeleton compared with natural human gait.

\begin{tabular}{|c|c|c|c|}
\hline Joint & $\begin{array}{c}\text { Degree of } \\
\text { freedom } \\
\text { (DOF) }\end{array}$ & Allowed & \multicolumn{2}{|c|}{ Exoskeleton design } \\
\hline Hip & 3 & Flexion/Extension & $\begin{array}{c}\text { abduction/adduction } \\
\text { Lateral/medial rotations }\end{array}$ \\
\hline Knee & 1 & Flexion/Extension & - \\
\hline Ankle & 3 & $\begin{array}{c}\text { No motions were } \\
\text { allowed }\end{array}$ & $\begin{array}{c}\text { Flexion/Extension } \\
\text { Inversion/Eversion } \\
\text { Lateral/medial rotations }\end{array}$ \\
\hline
\end{tabular}

As illustrated in figure 8 , the exoskeleton consists of a high torque DC servo motor mounted on a metal plate above the hip joint, and a link that transmits assistance torque from the motor to the thigh. The motor pushes or pulls the lower part of the thigh and creates moments at the hip joint These moments allow the hip to flex or extend. The High Torque DC Servo Motor is used for robots and other fields requiring high torque control. The most important features of the High 
Torque DC Servo Motor and the motor driver are summarized in table 2 . The microcontroller provides 14 digital input/output pins of which six can be used as pulse-width modulation (PWM) outputs, and six analog inputs; this can also be used as six digital input/output pins. It includes $5 \mathrm{v}$ linear regulator and a $16 \mathrm{MHz}$ crystal oscillator, a USB connection, a power jack, an ICSP header, and a reset button. The microcontroller can be powered by a USB cable or by an external battery.

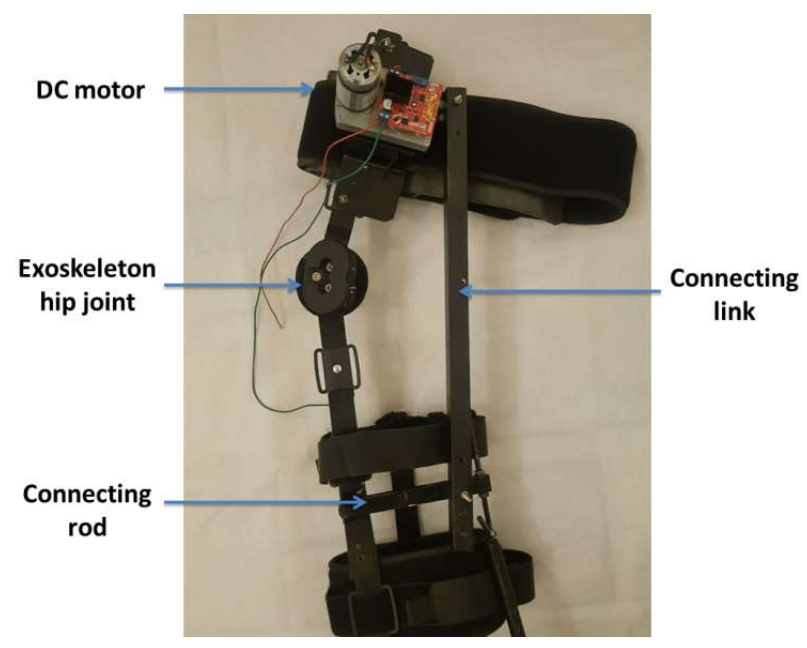

Fig. 8. Exoskeleton hip joint mechanism.

Table 2,

High torque DC servomotor and motor driver specifications

\begin{tabular}{|l|}
\hline Working voltage: DC12-24V \\
\hline No load current: $<500 \mathrm{~mA}$ \\
\hline Maximum torque value is $380 \mathrm{Kg} . \mathrm{cm}(24 \mathrm{v}) ; 1764 \mathrm{~N} . \mathrm{cm}(24 \mathrm{v}) ; 90 \mathrm{Kg} . \mathrm{cm}$ \\
$(12 v) ; 882 \mathrm{~N} . \mathrm{cm}(12 \mathrm{v})$. \\
\hline Angular velocity: $0.5 \mathrm{~s} / 60 \mathrm{6}$ egree \\
\hline Rotation angle: 330 degree MAX \\
\hline Input mode: PWMUN is PWM signal; ADIN is analog voltage signal \\
\hline Pulse signal input range: $0.5 \mathrm{~ms}-2.5 \mathrm{~ms}$ for all "multi-channel controller" \\
\hline Voltage signal input range: $0-5 \mathrm{~V}$ \\
\hline Control accuracy: 0.32 degree \\
\hline Weight: $530 \mathrm{~g}$ \\
\hline Size: $95.5 * 60.5 * 102.6 \mathrm{~mm}$ \\
\hline
\end{tabular}

The flexion/extension knee DOF was collocated with the human knee. The knee joint is passively actuated with a spring. The extended spring has a large stiffness value of $266.66 \mathrm{~N} / \mathrm{m}$, and it was $2 \mathrm{~cm}$ in diameter, and $20 \mathrm{~cm}$ in length. The knee joint mechanism is comprised of an extension spring in parallel to the leg as shown in figure 9 that is designed to operate with a tension load, so the spring stored energy during knee flexion and releases that energy to assist knee extension movements. This way, the spring is intended to store energy when the heel strikes the floor which is then released when the heel leaves the floor. The action of the passive component (spring) is combined with the mechanical output of the motor to provide a good control on the designed exoskeleton while walking.

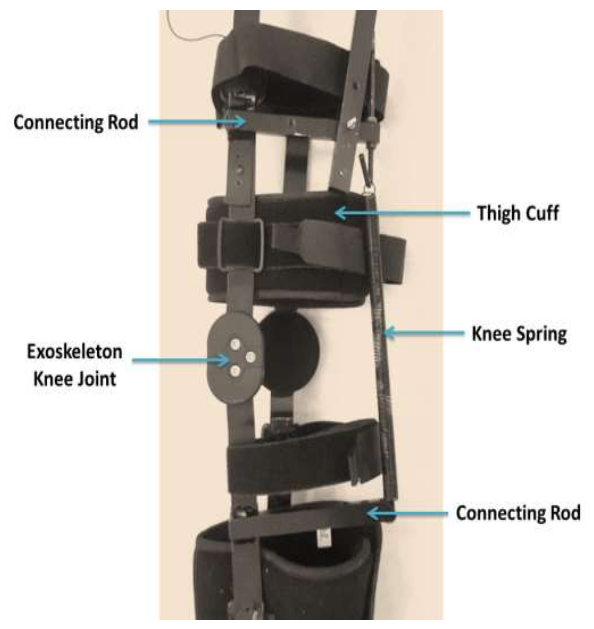

Fig. 9. Exoskeleton knee joint mechanism. 


\subsection{Electromyographic Activity}

The electromyography (EMG) of the right thigh and leg muscles were recorded and collected using myoresearch-xp clinical edition (1.07.01) standard EMG analysis protocols NORAXONUSA as illustrated in figure 10. The disposable, surface EMG electrodes are of type NORAXON-
USA [self-adhesive silver/silver chloride $(\mathrm{Ag} / \mathrm{AgCl})$ snap of dual electrodes $4 \mathrm{~mm}$ diameter electrode, used for surface EMG applications only]. The EMG activity was recorded from four muscles, these are: rectus femoris, biceps femoris, tibialis anterior, and the med. gastrocnemius muscles.

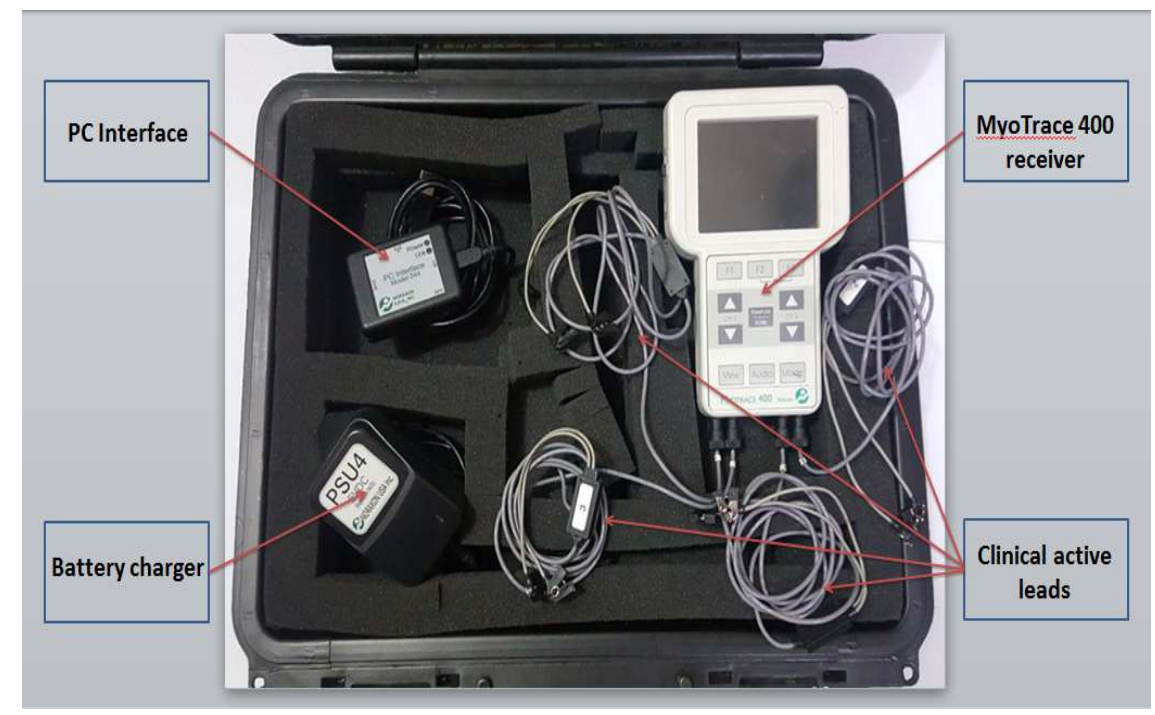

Fig.10. Noraxon EMG measurements device and its accessories.

\subsection{Clinical Evaluation}

The experimental tests on the sound person are implemented in order to obtain the behavior of gait cycle components, the lower extremity joints' angles and the activity of the lower limb muscles, this information is important in building technical data based on the gait cycle analysis of a healthy person. The obtained information is used in the analysis, comparison, and improvement indication in the design and manufacturing of the lower limb exoskeleton. Two subjects participated in this study, a healthy male participant with no history of neurological or muscular disease and no symptoms of orthopedic, and a subject with unilateral lower limb weakness. The participants were given a full explanation about the study, as well as a brief explanation about the function of the human lower limb muscles, and verbal instructions concerning the objectives and procedures of the research. Each participant gave informed consent before participating to the research. Some of the experimental tests are done in Biomechanics Laboratory in Biomedical Engineering Department at the faculty of engineering in Al-Nahrain University, while the others were in Biomedical Engineering Department at the faculty of Al-Khawarizmi College of Engineering, University of Baghdad. Experimental trials were conducted in order to verify the assist effect of the proposed exoskeleton. The main aim of data analysis and acquisition from trials was the comparison of the measured EMG activity while the subject was walking without and with the exoskeleton. The exoskeleton was adjusted to the subject's size. After that, the subject walked two or three practice trials to make sure the exoskeleton had a comfortable fit to the body and so that the subject should become familiar with the exoskeleton and training environment. Two different walking trials were recorded. After each trial the subject had a ten minute break. All trials were performed on a treadmill, as shown in figure 11, at fixed speeds; $0.8 \mathrm{~m} / \mathrm{s}$ for 3 minutes and $1.2 \mathrm{~m} / \mathrm{s}$ for 1 minute to simulate low to medium walking speed. The basic information of the participants and the different trials are summarized in tables 3 and 4 respectively. 


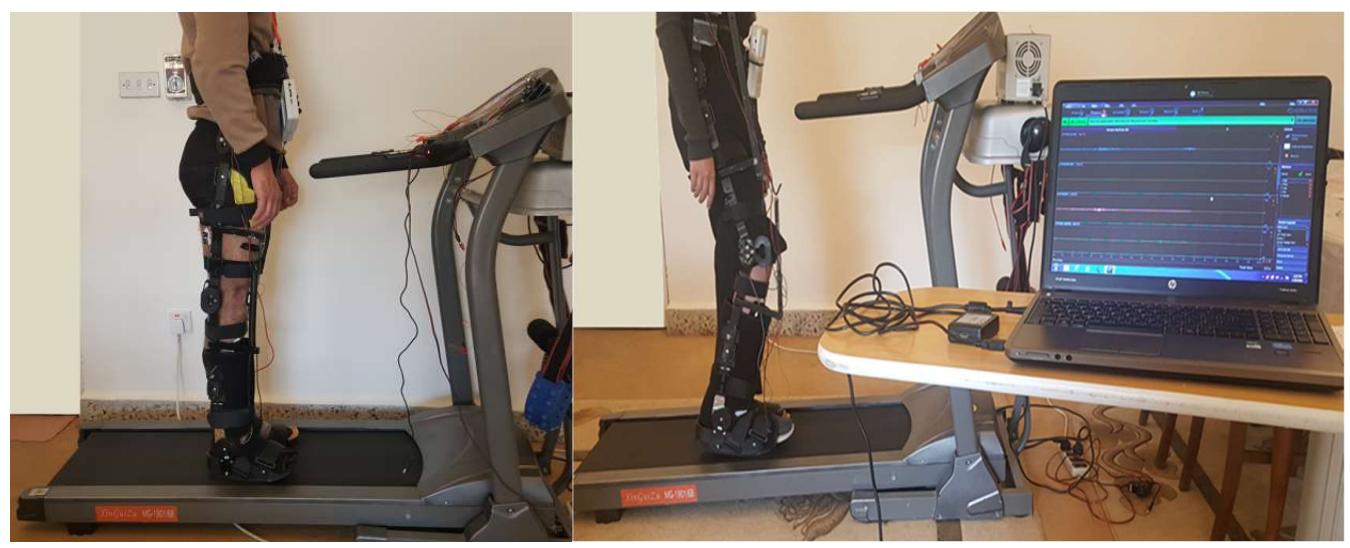

(a)

(b)

Fig.11. Experimental tests on participants (a) healthy subject (b) subject with unilateral (right) lower limb weakness.

Table 3,

Participants Basic information.

\begin{tabular}{|ccccc|}
\hline Participant condition & Age & Gender & $\begin{array}{c}\text { Body mass } \\
\text { (Kilogram) }\end{array}$ & $\begin{array}{c}\text { Body length } \\
\text { (meter) }\end{array}$ \\
\hline Healthy & 22 & Male & 63 & 1.7 \\
\hline Unilateral (right) lower limb weakness & 21 & Female & 59 & 1.67 \\
\hline
\end{tabular}

Table 4,

Walking conditions for sound person.

\begin{tabular}{|c|c|}
\hline Trial number & \multicolumn{1}{c}{ Waling Description } \\
\hline \hline $1^{\text {st }}$ trial & Walking without using the exoskeleton. \\
\hline $2^{\text {nd }}$ trial & Walking using the exoskeleton. \\
\hline
\end{tabular}

Throughout all trials, the EMG signals from four muscle groups on the right leg were recorded. The tested electrodes of each selected muscle are placed related to the learning picture equipped with the NORAXON-USA software. Signals from the EMG electrodes were transmitted to the special purpose software to be recorded and analyzed. The raw EMG signals were filtered using a notch filter $(50 \mathrm{~Hz})$ to minimize the effects of the alternating current. Then, a band-pass filter $(80-250 \mathrm{~Hz})$ was used to remove motion artifacts. The EMG signals were then rectified and the EMG mean values were obtained.

\section{Results and Discussion}

The results of EMG activity of the right leg muscles of the healthy subject while walking on the treadmill at fixed speeds of $0.8 \mathrm{~m} / \mathrm{s}$ for 3 minutes and $1.2 \mathrm{~m} / \mathrm{s}$ for 1 minute are presented in figures 12 and 13, respectively. The figures reveal the mean EMG activity of the med. gastrocnemius, tibialis anterior, biceps femoris, and rectus femoris muscles while walking without and with the exoskeleton. Walking using the proposed exoskeleton has an effect on muscle (EMG) activation, when compared to free walking (walking without the exoskeleton). The exoskeleton has different impact on each muscle depends on the location and tendons of these muscles. The four muscles' efforts on both the front and the back sides of the user's leg were decreased when walking using the exoskeleton. The motor supports and drives the leg through the appropriate step pattern during the gait cycle by adding power at the hip joint, while the knee flexion/extension has spring loaded which provides an extension torque and assists knee movements resulting in a strong effect on the leg muscles which contribute in knee flexion/extension when walking. The output of the four leg muscles was decreased significantly. This indicates less muscle effort is required to hold the 
limb while walking. The most affected muscles were the med. gastrocnemius and the rectus femoris muscle which were decreased by $47.17 \%$ and $45.95 \%$, respectively. As the contraction of the rectus femoris muscle contributes to hip flexion as well as knee extension, and contraction of the med. gastrocnemius muscle contributes to knee flexion, it is not surprising that the muscle activity is down when walking using the entire exoskeleton with the motor and spring installed.

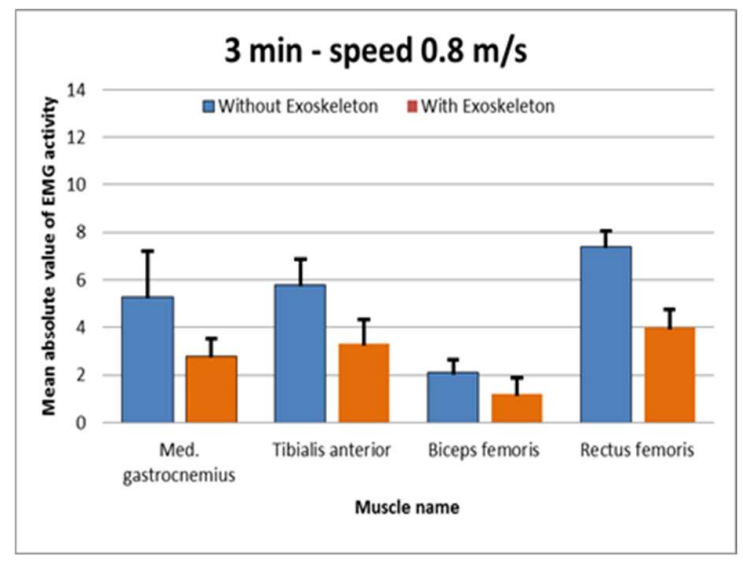

Fig. 12. Mean EMG activities of the right leg muscles of the healthy subject during walking on treadmill at fixed speed $0.8 \mathrm{~m} / \mathrm{s}$ for 3 minutes without and with the exoskeleton.

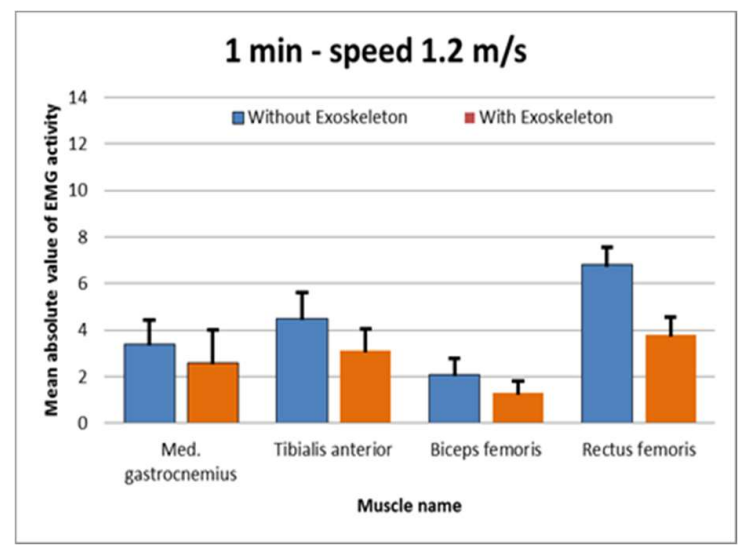

Fig.13: Mean EMG activities of the right leg muscles of the healthy subject during walking on treadmill at fixed speed $1.2 \mathrm{~m} / \mathrm{s}$ for 1 minute without and with the exoskeleton.

The EMG activity tests are applied on the subject with unilateral lower limb weakness while walking on a treadmill at two fixed walking speeds $0.8 \mathrm{~m} / \mathrm{s}$ and $1.2 \mathrm{~m} / \mathrm{s}$ for 3 and 1 minutes, respectively. The EMG signals of the muscles contributed in the motion of the leg were collected and analyzed. The mean EMG signals when walking without and with the exoskeleton are shown in figure 14 and 15 . The four muscles' efforts of the patient's leg were decreased when walking on a treadmill for 3 and 1 minutes at two fixed speeds $0.8 \mathrm{~m} / \mathrm{s}$ and $1.2 \mathrm{~m} / \mathrm{s}$ using the entire exoskeleton with the motor and spring installation. The motor supports the patient's leg and drives it through appropriate step pattern while walking by providing torque at the hip joint, while the knee flexion/extension was spring loaded which provides an extension torque and assists knee movements resulting in an effect on the leg muscles which are contributed in knee flexion/extension while walking. The reduction in EMG signals indicates less muscle effort is required by the patient to hold the limb while walking using the exoskeleton. The most affected muscles were the rectus femoris and tibialis anterior on the front sides of the user's leg which are decreased by $20.755 \%$, and $29.41 \%$, when walking at $0.8 \mathrm{~m} / \mathrm{s}$ for 3 minutes, and $38.89 \%$, and $28.1 \%$, when walking at $1.2 \mathrm{~m} / \mathrm{s}$ for 1 minute, respectively. As contraction of the rectus femoris muscle contributes to hip flexion as well as knee extension, it is not surprising that the muscle activity is down when walking using the entire exoskeleton with motor and spring installation. Providing adequate foot clearance during the swing of the limb when walking with the entire exoskeleton can be a reason for decreasing in EMG signals of the tibialis anterior muscle.

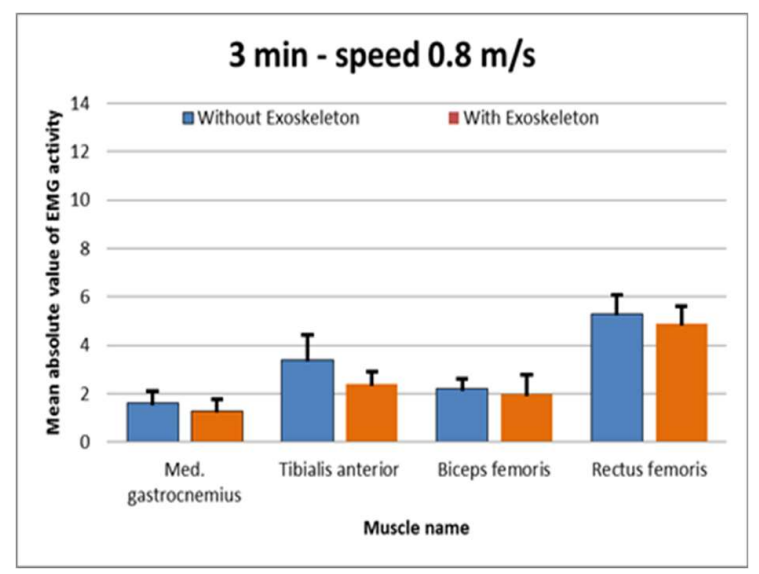

Fig. 14. Mean EMG activity of the right leg muscles of the subject with lower limb weakness during walking on treadmill at fixed speed $0.8 \mathrm{~m} / \mathrm{s}$ for 3 minutes. 


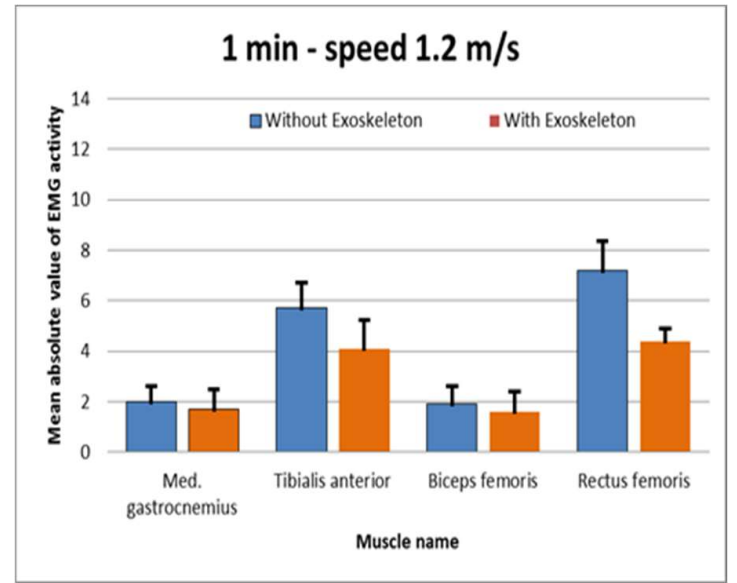

Fig. 15. Mean EMG activity of the right leg muscles of the subject with lower limb weakness during walking on treadmill at fixed speed $1.2 \mathrm{~m} / \mathrm{s}$ for 1 minute.

\section{Conclusion}

Quasi-passive lower limb exoskeletons usually employ a reduced number of actuators and require relatively a straight forward control strategy, resulting in energy efficient, lightweight, and operationally quiet lower limb devices. In this paper the analysis and development of a 2 DOFs, autonomous, lightweight, and quasi-passive lower limb exoskeleton was presented to improve the human gait cycle. The linkage type selection is justified. A mathematical model of the human gait cycle was developed for calculating the torques at the hip joint. The mathematical model was based on the theoretical analysis of the human extremity limb throughout a full gait cycle consisting of both the stance and swing phases and the gait subphases, by investigating the behavior of the lower limb joints, the length and weights of the lower limb components, and the GRFs. For the proposed exoskeleton, the spring in combination with the motor can support and assist the user's lower limb to walk in an appropriate step pattern. The results showed that the presented exoskeleton reduces lower limb muscles' effort for both the healthy and disabled subjects, as an indication that the proposed exoskeleton can be used as an assistive device to give better mobility for both healthy and disabled people. For further improvements of the current quasi-passive exoskeleton for future applications in rehabilitation fields, efforts should be concentrated on the types of materials, actuators, controllers, energy efficient, safety, and cost effectiveness of the exoskeletons.

\section{References}

[1] G. M. Cestari, D. Sanz-Merodio, F.C. Arevalo, E, "ARES, a variable stiffness actuator with embedded force sensor for the ATLAS exoskeleton." Industrial Robot: An International Journal, pp. 518-526, 2014.

[2] K. Kong and D. Jeon, "Design and control of an exoskeleton for the elderly and patients," IEEE/ASME Trans. Mechatronics, vol. 11, no. 4, pp. 428-432, 2006, doi: 10.1109/TMECH.2006.878550.

[3] M. D. C. Sanchez-Villamañan, J. GonzalezVargas, D. Torricelli, J. C. Moreno, and J. L. Pons, "Compliant lower limb exoskeletons: A comprehensive review on mechanical design principles," J. Neuroeng. Rehabil., vol. 16, no. 1, pp. 1-16, 2019, doi: 10.1186/s12984-019-0517-9.

[4] R. Stopforth, "Customizable rehabilitation lower limb exoskeleton system," Int. J. Adv. Robot. Syst., vol. 9, pp. 1-7, 2012, doi: $10.5772 / 53087$.

[5] S. Krut, M. Benoit, E. Dombre, and F. Pierrot, "MoonWalker, a lower limb exoskeleton able to sustain bodyweight using a passive force balancer," Proc. - IEEE Int. Conf. Robot. Autom., pp. 2215-2220, 2010, doi: 10.1109/ROBOT.2010.5509961.

[6] M. Lyu, W. Chen, X. Ding, J. Wang, S. Bai, and H. Ren, "Design of a biologically inspired lower limb exoskeleton for human gait rehabilitation," Review of Scientific Instruments, vol. 87, no. 10. American Institute of Physics Inc., 01-Oct-2016, doi: 10.1063/1.4964136.

[7] W. Huo, S. Mohammed, Y. Amirat, and K. Kong, "Active Impedance Control of a lower limb exoskeleton to assist sit-to-stand movement," Proc. - IEEE Int. Conf. Robot. Autom., vol. 2016-June, pp. 3530-3536, 2016, doi: 10.1109/ICRA.2016.7487534.

[8] B. Chen et al., "Recent developments and challenges of lower extremity exoskeletons," J. Orthop. Transl., vol. 5, pp. 26-37, 2016, doi: 10.1016/j.jot.2015.09.007.

[9] G. Chen, C. K. Chan, Z. Guo, and H. Yu, "A review of lower extremity assistive robotic exoskeletons in rehabilitation therapy," Crit. Rev. Biomed. Eng., vol. 41, no. 4-5, pp. 343-363, 2013, doi: 10.1615/CritRevBiomedEng.2014010453.

[10] J. Zhou, S. Yang, and Q. Xue, "Lower limb rehabilitation exoskeleton robot: A review," vol. 13, no. 1038, pp. 1-17, 2021, doi: 
$10.1177 / 16878140211011862$.

[11] T. Mikolajczyk et al., "Advanced technology for gait rehabilitation: An overview," Adv. Mech. Eng., vol. 10, no. 7, pp. 1-19, 2018, doi: $10.1177 / 1687814018783627$.

[12] N. K. Al-hayali, J. S. Chiad, S. M. Nacy, and O. Hussein, "A Review of Passive and Quasi-Passive Lower Limb Exoskeletons for Gait Rehabilitation," vol. 44, no. 9, pp. 428439, 2021.

[13] H. Kawamoto, S. Lee, S. Kanbe, and Y. Sankai, "Power assist method for HAL-3 using EMG-based feedback controller," Proc. IEEE Int. Conf. Syst. Man Cybern., vol. 2, pp. 1648-1653, 2003, doi: 10.1109/icsmc.2003.1244649.

[14] Y. He, D. Eguren, T. P. Luu, and J. L. Contreras-Vidal, "Risk management and regulations for lower limb medical exoskeletons: A review," Med. Devices Evid. Res., vol. 10, pp. 89-107, 2017, doi: 10.2147/mder.s107134.

[15] X. Zhang, Z. Yue, and J. Wang, "Robotics in Lower-Limb Rehabilitation after Stroke," Behav. Neurol., vol. 2017, 2017, doi: 10.1155/2017/3731802.

[16] Z. Yang, W. Gu, J. Zhang, and L. Gui, Force Control Theory and Method of Human Load Carrying Exoskeleton Suit. 2017.

[17] C. Woods, L. Callagher, and T. Jaffray, "Walk tall: The story of Rex Bionics," J. Manag. Organ., no. December, pp. 1-14, 2018, doi: 10.1017/jmo.2018.68.

[18] G. Barbareschi, R. Richards, M. Thornton, T. Carlson, and C. Holloway, "Statically vs dynamically balanced gait: Analysis of a robotic exoskeleton compared with a human," Proc. Annu. Int. Conf. IEEE Eng. Med. Biol. Soc. EMBS, vol. 2015-Novem, no. September, pp. 6728-6731, 2015, doi: 10.1109/EMBC.2015.7319937.

[19] V. Lajeunesse, C. Vincent, F. Routhier, E. Careau, and F. Michaud, "Exoskeletons' design and usefulness evidence according to a systematic review of lower limb exoskeletons used for functional mobility by people with spinal cord injury," Disabil. Rehabil. Assist. Technol., vol. 11, no. 7, pp. 535-547, 2016, doi: 10.3109/17483107.2015.1080766.

[20] S. K. Banala, S. K. Agrawal, and J. P. Scholz, "Active Leg Exoskeleton (ALEX) for gait rehabilitation of motor-impaired patients," in 2007 IEEE 10th International Conference on Rehabilitation Robotics, ICORR'07, 2007, pp. 401-407, doi: 10.1109/ICORR.2007.4428456.

[21] S. K. Banala, S. K. Agrawal, S. H. Kim, and J. P. Scholz, "Novel gait adaptation and neuromotor training results using an active leg exoskeleton," IEEE/ASME Trans. Mechatronics, vol. 15, no. 2, pp. 216-225, 2010, doi: 10.1109/TMECH.2010.2041245.

[22] S. K. Banala, S. H. Kim, S. K. Agrawal, and J. P. Scholz, "Robot assisted gait training with active leg exoskeleton (ALEX)," Proc. 2nd Bienn. IEEE/RASEMBS Int. Conf. Biomed. Robot. Biomechatronics, BioRob 2008, vol. 17, no. 1, pp. 653-658, 2008, doi: 10.1109/BIOROB.2008.4762885.

[23] "Ekso Bionics." [Online]. Available: https://eksobionics.com/[accessed 04.10.15]. [Accessed: 05-Feb-2020].

[24] Y. M. Pirjade, D. R. Londhe, N. M. Patwardhan, A. U. Kotkar, T. P. Shelke, and S. S. Ohol, "Design and Fabrication of a Low-cost Human Body Lower Limb Exoskeleton," 2020 6th Int. Conf. Mechatronics Robot. Eng. ICMRE 2020, pp. 32-37, 2020, doi: 10.1109/ICMRE49073.2020.9065128.

[25] M. Cardona and C. E. García Cena, "Biomechanical Analysis of the Lower Limb: A Full-Body Musculoskeletal Model for Muscle-Driven Simulation," IEEE Access, vol. 7, pp. 92709-92723, 2019 , doi: 10.1109/ACCESS.2019.2927515. 


\title{
تحليل وتقييم الهيكل الخارجي شبه السلبي المصمم للطرف السفلي لغرض إعادة تأهيل المشي
}

\author{
سومر متي ناسي عزي

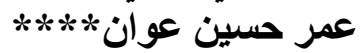

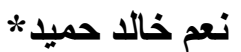

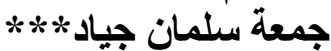

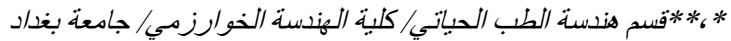

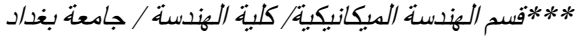

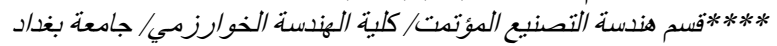

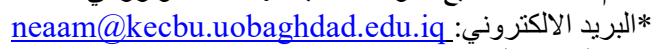 \\ somernacy@googlemail.com:البريد الالكتروني:***روني

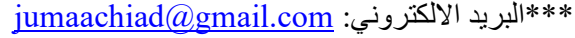

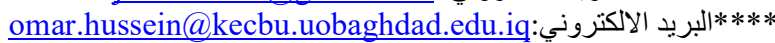

الخلاصة

من الممكن أن يساعد استخدام الهياكل الخارجية المصممة للطرف السفلي في أداء وظائف معينة كالمساعدة على المشي، ودعم العلاج الطبيعي

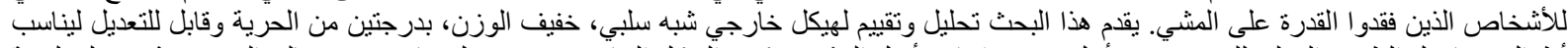

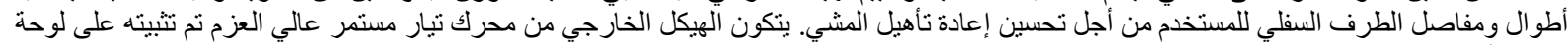

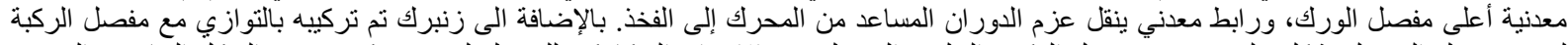

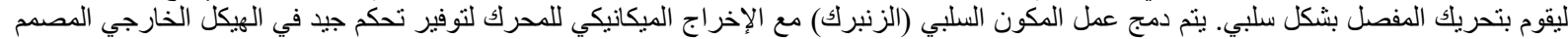

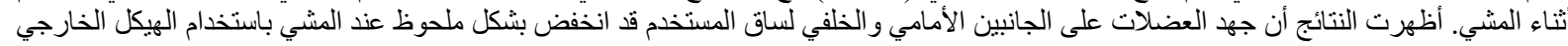
الذي تم تصميمه للطرف السفلي. 